\title{
eStorys: A visual storyboard system supporting back-channel communication for emergencies is $^{2}$
}

\author{
A. Malizia ${ }^{\mathrm{a}, *}$, A. Bellucci ${ }^{\mathrm{a}}$, P. Diaz ${ }^{\mathrm{a}}$, I. Aedo ${ }^{\mathrm{a}}$, S. Levialdi ${ }^{\mathrm{b}}$ \\ a Department of Computer Science, Universidad Carlos III de Madrid, Spain \\ ${ }^{\mathrm{b}}$ Department of Computer Science, Sapienza Universitá di Roma, Italy
}

Keywords:

Mashups

Collaboration systems

Emergency management

\begin{abstract}
A B S T R A C T
In this paper we present a new web mashup system for helping people and profes sionals to retrieve information about emergencies and disasters. Today, the use of the web during emergencies, is confirmed by the employment of systems like Flickr, Twitter or Facebook as demonstrated in the cases of Hurricane Katrina, the July 7, 2005 London bombings, and the April 16, 2007 shootings at Virginia Polytechnic University. Many pieces of information are currently available on the web that can be useful for emergency purposes and range from messages on forums and blogs to georeferenced photos. We present here a system that, by mixing information available on the web, is able to help both people and emergency professionals in rapidly obtaining data on emergency situations by using multiple web channels. In this paper we introduce a visual system, providing a combination of tools that demonstrated to be effective in such emergency situations, such as spatio/temporal search features, recommendation and filtering tools, and storyboards. We demonstrated the efficacy of our system by means of an analytic evaluation (comparing it with others available on the web), an usability evaluation made by expert users (students adequately trained) and an experimental evaluation with 34 participants.
\end{abstract}

\section{Introduction}

Hazards and disasters happen. Think of terrorist attacks such as the 9/11 (the suicide attacks by al Qaeda on the United States Twin Towers) or the equally sadly known train bombing at the Atocha station in Madrid (11 March 2004) in which hundreds of people lost their lives or were wounded. Natural disasters such as wildfires, hurricanes, tornadoes or tsunamis destroy everything they encounter and leave people without resources and completely overwhelmed. Emergency management aims at such large scale events.

\footnotetext{
This paper has been recommended for acceptance by S.-K. Chang.

* Corresponding author.

E-mail addresses: amalizia@inf.uc3m.es (A. Malizia), abellucc@inf.uc3m.es (A. Bellucci),pdp@inf.uc3m.es (P. Diaz), aedo@ia.uc3m.es (I. Aedo), levialdi@di.uniroma1.it (S. Levialdi).
}

The multitude of natural and human made disasters we have to face in modern society provides more than enough reasons to justify the governments' efforts for the introduction of agencies addressing emergency situations. However, emergency management planning is not solely a governmental responsibility, but a community activity [27]. At the beginning, every emergency situation, regard less of its entity and extension, is a local event, and local actors firstly deal with the disaster. Palen et al. [25] stated that most of the time ordinary people, for example single or organized in volunteer groups, providing help during a crisis situation by performing useful activities such as rescue people in their houses, communicate and report to authorities, etc. Non governmental public's participation in disaster management demonstrates how significant is the work performed even outside the official response efforts $[12,30,11]$. 
During or immediately after an emergency there is a huge number of social interactions taking place: people communicating the emergency status with others, damages evaluation, information request about relatives, and so on. With the advent of Internet and of social network services [10], non official back channel commu nications [29] became widespread since people are taking advantage of the existing communication technologies by organizing life saving activities among each other, inde pendently, or in parallel with, official national emergency management channels. We refer to [29] for the definition of back channel as an unofficial communication channel between various entities used to supplement official channels.

The growing presence of communications technology, new media and digital devices, in fact, is making public participation more tangible during emergencies. As an example, the proliferation of photo capture devices, such as digital cameras or mobile phones with an integrated camera, has enabled grassroots journalism [13], allowing first responders and people present to visually document a disaster situation as it is happening. A clear example as been described in [23], where the case of 2005 London bombings is presented together with the use of Flickr, ${ }^{1} \mathrm{a}$ photo sharing web service, for creating groups on bomb ings topics (such as the London Bomb Blast Community). These groups shared pictures on the London bombings asking users for posting all the personal photos they had on the bombing sites before and after the accident, in order to inform the world. Moreover, web services like Flickr, permitting users to store, share and retrieve pictor ial content, inspire new forms of communications and self organization during disaster response by viewing the photo sharing activity as a form of social media.

During crisis management activities a huge amount of data from heterogeneous sources is generated: pictorial and video feeds, news reports, e mail and text messaging. Most of this data expose geospatial information (i.e. associated metadata) or implicit location references (i.e. the name of a place in a news report). In such a scenario, geocollaboration bears on people working together to solve a geospatial problem taking into account georeferenced data, as described in [27]. So, geography plays an important role in emergency management and a visual representation makes this information tangible and useful.

We present here a novel collaborative mapping mashup, enabling users to visualize, edit and share georeferenced media content, according to spatial temporal features. We refer to the mashup as a web application, combining data and services from different existing systems, into a single integrated tool. Our mashup application gathers pictures (and associated metadata like keywords and tags added by users) from the Flickr online database and employs location metadata to place them on a map. Temporal metadata are considered, providing an interface for efficiently browse large user contributed georeferenced media collections. Despite the existence of different map based photo brows ing online services, to our knowledge, our contribution

\footnotetext{
${ }^{1}$ www.flickr.com
}

represents a first effort in combining storyboards with the spatial and temporal dimensions for media retrieval and browsing in such mashup applications. Our main goal is to use the explicitly disclosed location metadata (latitude and longitude) as well as the temporal one (i.e. at what time a photo was taken) to enable users to quickly retrieve photos of a certain place over a certain temporal interval (one day, one week or one month). Moreover, we believe that temporal information in conjunction with locations can be valuable in enhancing geocollaboration. The use of spatio temporal dimensions has been combined with tools enabling the combination of such dimensions. We identified four dimen sions and designed the corresponding tools, for managing these media collections available on the web. These four dimensions are spatial (latitude and longitude), temporal (date and temporal intervals), social (recommendation and collaborative filtering) and situational (storyboards). We show here a collaborative storyboard authoring tool, allowing the user to easily generate and share spatial and temporal photos' sequences exploiting the drag and drop of selected images. Lastly, our application supports social navigation, in the sense that users' past interactions with the system are employed as recommendations, impacting on the way the information is presented during other users' interactions.

The key contribution of our work is to show how current web social media, technologies and services, together with the presence of a huge amount of georeferenced materials over the web, can be easily and successfully exploited to create new geocollaboration tools enabling back channel communications during disaster situations. In the next sections we will describe the preliminary studies we made, the system we developed and the experimental evaluation we conducted. In particular in Section 2, we review litera ture and systems comparable to our approach. In the successive Section 3, we describe the system we present here and the designing choices together with implementa tion. Experiment results are reported in Section 4, where we conducted three different evaluations: one analytic, one heuristic evaluation with experts and one experimental evaluation with 34 participants. Section 5 is about discus sions and conclusions on our research, while Appendices A and $B$ present the evaluation tool (questionnaire design and final implementation).

\section{Background}

In this section we describe the literature and the research we conducted on existing systems and approaches in two main aspects related to our system: back channel communications (in the emergency systems domain) and geospatial Web paradigm together with mapping mashups. Furthermore, we present a classification of existing and reviewed mashup systems based on a set of design dimensions we identified by carefully reviewing the corre spondent literature. We restricted our classification to map based mashup systems. We think that there are many media contents available on the web through different social networks which are not integrated to provide users with an overall view of georeferenced information during emergency situations. Georeferenced information during such emergencies is crucial for a rapid understanding of 
emergency status, recovery plans, providing local informa tion about damages, etc. Since georeferenced data are complex and require integration of different contents on a map specific tools are required to manage such data as stated in [32]. For the above reasons we focused on mashup systems based on maps providing tools for search and navigate information placed on maps.

\subsection{Back channel communications in emergency management}

In times of emergency, members of the public tend to improvise and perform various activities, such as provide first aid to wounded people, victims transportation to hospitals or even take photos to document the event $[25,23]$. Along with these activities, taking place physi cally on the disaster area, a huge number of social interaction among citizens occur. In a disaster situation people need information. They seek it for themselves and, at the same time, try to provide helpful information, such as the emergency status or damages evaluation, to other citizens, including their relatives or friends. This phenom enon is often ignored by the members of governmental agencies, which are almost entirely focused on their official role in the process of dealing with the disaster. Therefore, in such a context, people communications are considered back channels (or peer to peer) activities, in contrast with the information provided by the official channels [29]. Although back channel communications can be viewed, in the emergency management domain, as potential vehicles to spread misinformation and rumours compromising the public safety, their presence is growing with each new disaster.

During emergencies, online social media are increasingly gaining prominence for the members of the public to find and provide information independently, or in parallel, with official channels. Social services, such as collaborative tag ging systems, social networking sites or even blogs and wikis, support peer to peer communications. Such systems allow users to both produce and consume information about the disaster. In this way citizens can organize among themselves and share information exploiting existing tech nologies. This fact clearly shows how the presence of information and communications technology is changing the disaster response arena, making back channel commu nications and people involvement more tangible [29].

\subsubsection{Social media and open source software enabling back channels communication}

The most common type of online activity consists of finding and sharing information about personal property, relatives and friends safety and sources of relief. As an example, during the 2007 wildfire disaster in California, Twitter $^{2}$ was employed by local citizens and organizations to provide updates about the fires situation in the region. Twitter is a blogging service allowing users to send text messaging posts to the Twitter website. Posts are instantly delivered to the mobile phone or computer of

\footnotetext{
${ }^{2}$ www.twitter.com
}

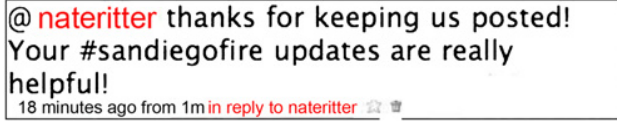

Fig. 1. Twitter hash tags.

other users who have signed up to receive them. Users can also add metadata to their tweets, in the form of hashtags, by prefixing a keyword with a hash symbol: during the 2007 forest fires a twitter user $^{3}$ used the hashtag "\#sandiegofire" to identify his updates, helping people in acquiring useful information related to the disaster (Fig. 1).

Another example during the same emergency situation was the one provided by the use of Google Maps: people created and annotated maps with markers indicating burnt areas, evacuation areas, shelters, schools and closed down businesses. One of the most popular maps was created and maintained by KPBS news, which received more than 1.7 million views over the course of the firestorm [29].

E mail, Instant Messaging tools and social networking systems like Facebook ${ }^{4}$ can be used to trace online users activities and to determine whether people are safe or not. For instance, IM informs on the online status of a user telling us if she is currently connected, is typing on the keyboard or is away from the computer. Facebook is a website allowing users to connect and interact with other people.Users can add friends and send them messages, and update their personal profile to notify friends about themselves. As reported in [15] users could deduce relatives or friends current condition by simply interpret ing their signs of activity on the website inferring, for example, that a friend is OK because she just posted a message on her Facebook account.

Facebook, for instance, was used during the shooting at Virginia Tech in April 2007, by students to provide and share critical information and activities going on at the campus, informing quickly on the casualties and injuries through the Facebook social network [23].

\subsection{Geospatial web and mapping mashups}

Geospatial Web (or GeoWeb) is a term identifying a new paradigm to access and explore data on the web allowing users to navigate, access, and visualize georefer enced data as they would in a physical world [19]. Merging location based information with the content currently available on the web creates an environment, where things can be searched using location metadata instead of employing only keywords. As a result, in the last few years, thanks to the increase of web development meth ods (e.g. AJAX Asynchronous Javascript And XML) and the efforts in defining standards protocols for content definition and exchange such as SOAP, ${ }^{5}$ and RSS, ${ }^{6}$ we are

\footnotetext{
${ }^{3}$ Nate Ritter.

${ }^{4}$ www.facebook.com

5 http://www.w3.org/TR/soap/

${ }^{6}$ http://cyber.law.harvard.edu/rss/rss.html
} 
witnessing in a proliferation of web applications allowing users to directly search, create, modify and share online maps. Web maps are increasingly becoming a place, where knowledge and meanings can be traced and visua lized: current web mapping services like Google Maps, ${ }^{7}$ Google Earth ${ }^{8}$ and Yahoo! Maps, ${ }^{9}$ for example, provide features enabling users to quickly create and share cus tomized 2D and 3D maps with relatives or friends. With Google Maps users can create their own maps adding place markers, shapes or lines defining locations or paths. Furthermore, cartographic data can be annotated with georeferenced multimedia content such as images or videos. At this stage the potential of connecting multi media content over the web through locations metadata has become straightforward. Through simple Application Programming Interfaces (APIs), made available by the different web services, designers can easily develop web mapping mashups exploiting the synergy of different data sources, integrating a variety of content (such as images) into an existing digital map. One of the most clear examples of a mapping mashup can be the ChicagoCri me.org website which integrates crime data from the Chicago Police Department's database with cartographic data from Google Maps. Another simple example is the Hurricane Digital Memory Bank ${ }^{10}$ website, a project to collect and share the users' digital contribution on the hurricanes Katrina and Rita.

At the current time a huge amount of georeferenced content is accessible over the web, including geographically annotated web pages, blogs, digital photographs and videos. In particular, considering the image media, the increase of digital photo capture devices and the growing users' atti tude in sharing their personal photographs has led to the creation of large community contributed pictures collec tions available online. As stated in [31], we can identify at least six different ways to acquire location metadata for image media which include manual entry as well as the employment of location aware camera phones and digital cameras or GPS devices. According to [17] location informa tion such as geographic coordinates, associated to images, can help in automatically understanding photo's semantics, as well as browsing and organizing photos collections. Collaborative systems enabling users to publish and share photographs they own, like Flickr, currently host billions of images with associated metadata such as who took the picture, where and when it was taken and, of course, tags inserted by the user, describing the picture content.

Therefore, the Geospatial Web paradigm in conjunc tion with available media collections offers to mashups designer the possibility to create new collaborative map ping applications simply aggregating pictures, associated metadata and cartographic content. Efforts in this direc tion started in 2001, in [31] the authors describe WWMX, a map based system to browse and visualize on a map a collection of georeferenced photos. Nevertheless, this system has not been update since time and it is a standalone application. In [2] the authors analyse the tags associated with georeferenced Flickr images to find repre sentative tags for arbitrary areas in the world, using a map interface to display the derived tags and the original photo items (see Fig. 3). Other recent examples of map based photo browsing systems are Flickr Map ${ }^{11}$ and Google's Panoramio. ${ }^{12}$ Although both these systems could represent and interesting approach to map mashup the main limitation consists of reduced browsing capabilities. Considering the combination of spatio temporal features to manage georeferenced information, the two mashups http://earthquakes.googlemashups.com/ and http://earth quakes.tafoni.net/ are noteworthy. These two systems receive notifications about earthquakes from different news services and localize them on a map, in a temporal order. Users can read news (as well as read blog entries or view video related) to a particular earthquake. As the mashups are directly connected with the U.S. Geological Survey Earthquake Hazards Program (http://earthquake. usgs.gov/), users can also insert their report regarding their own experience. These systems can only be used to visualize earthquake news but spatio temporal searching features are not included.

Another interesting example on how the GeoWeb para digm can be successfully applied in the field of emergency management is the one offered by the Ushahidi ${ }^{13}$ platform. Ushahidi (testimony in Swahili), is essentially an open source project aims at gathering user generated crisis infor mation, allowing anyone to submit content through text messaging using a mobile phone, e mail or web form. The project born as a simple website mashup created to report on the post election violence in Kenya (February 2008), using user generated reports and Google Maps. After that, the Ushahidi engine was employed in a variety of crisis situation; for example, the Arabic language news network Al Jazeera uses Ushahidi in their War on Gaza ${ }^{14}$ website to cover the activity happening in Gaza in January 2009 (see Fig. 2). With the Ushahidi mashup, users can submit their reports about the event, assigning them a name, a brief description, a date, a category (within predefined ones) and a location. In this way, the system can place the report on the map, providing to the users an interface to browse within different reports by click on the dots on the map and filtering employing the different categories. An overview of reported incidents over time is offered, giving the possibility to filter and visualize events within selected temporal intervals. Nevertheless this system is highly customized depending on the scenario selected by the mashup designer. For this reason it is not applicable in general scenarios but a specific mashup application should be developed case by case, and thus providing the functionalities chosen by the designer according to the specific situation. Even Sahana, ${ }^{15} \mathrm{a}$ web based collaboration tool that addresses the common coordination problems during a disaster [9], has recently

\footnotetext{
11 flickr.com/map

12 www.panoramio.com

13 www.ushahidi.com

14 http://labs.aljazeera.net/warongaza/

15 http://www.sahana.lk/
}

\footnotetext{
7 maps.google.com

8 earth.google.com

9 maps.yahoo.com

${ }^{10}$ hurricanearchive.org/map
} 


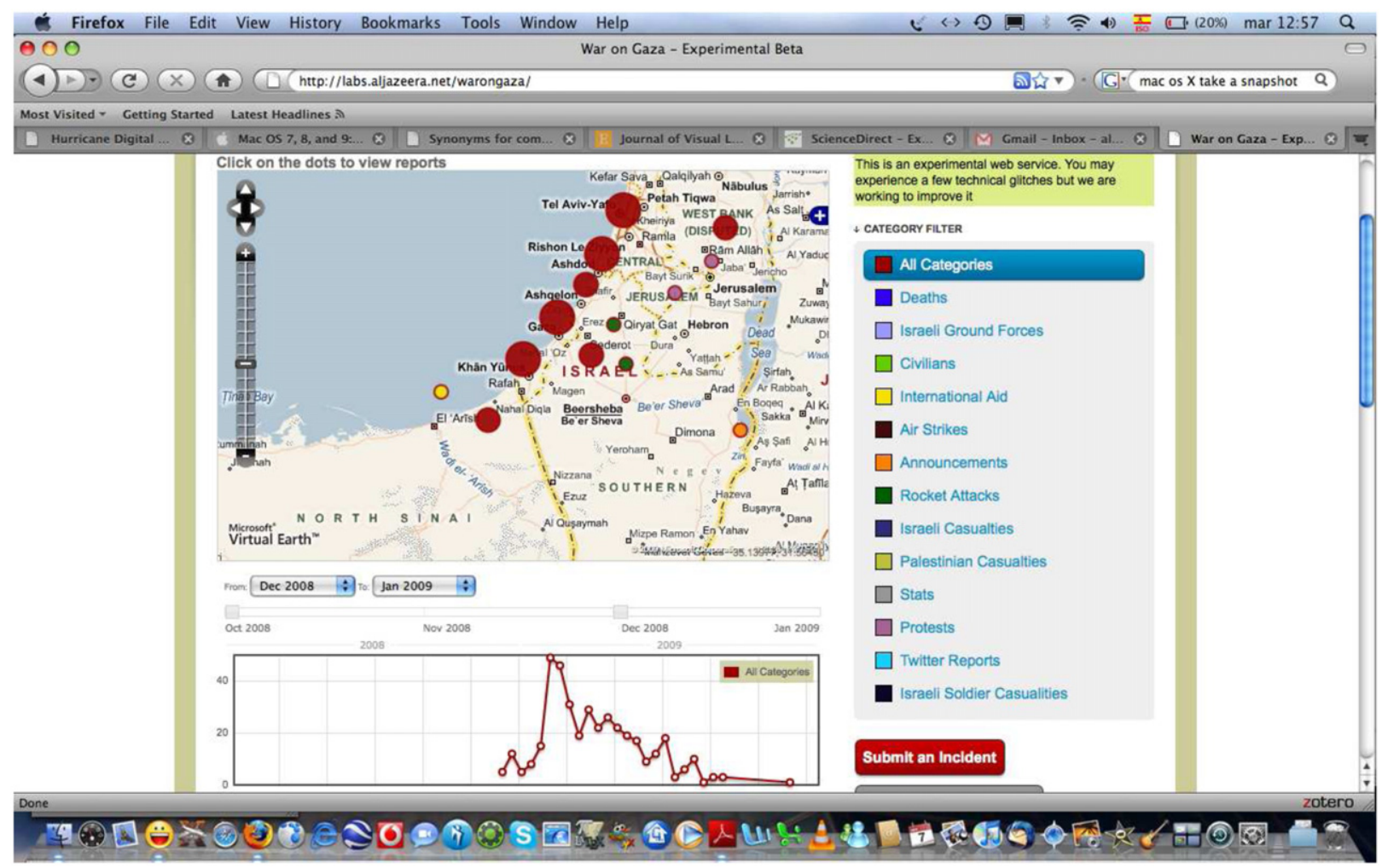

Fig. 2. The War on Gaza website built employed the Ushahidi engine.

integrated Google Maps in order to provide a GIS (Geogra phical Information System) view of affected regions.

Note that mashups rely on standards (SOAP, REST, ${ }^{16}$ RSS, JSON ${ }^{17}$ ), since only standards protocols allow easy adapta tion of content according to the change of context. There fore, mashup frameworks as well as mashup editors (Yahoo Pipes $^{18}$ see Fig. 4, Google Mashup Editors ${ }^{19}$ ) have recently become very popular, allowing users to easily create their mashup application regardless of their technical skill level.

\subsubsection{Mashups classification by designing dimensions}

By studying and exploring the existing literature con cerning geospatial Web, mapping mashups, and the use of such systems during emergencies we identified four dimen sions. We used these four dimensions in order to categorize web applications for managing spatio/temporal, georefer enced and user contributed media collections available on the web. The four dimensions are: spatial (geographic information), temporal (navigation over date and time), collaborative (collaborative features) and situational. We would like to emphasize here the situational aspect. In particular, there are two aspects of situational elements that are part of such systems: situational designers and

\footnotetext{
${ }^{16}$ http://www.ics.uci.edu/ fielding/pubs/dissertation/rest_arch_ style.htm

17 http://www.json.org

18 pipes.yahoo.com/pipes/

19 code.google.com/gme/
}

situational contributors. In fact, mashup applications are, generally, designed by situational designers to extract infor mation for their own use from collaborative systems such as Flickr, Del.icio.us, Technorati, etc. By situational designer we mean a person developing an application for his/her perso nal use that can be shared over the web to be used by others having the same needs. Such systems are usually built by mashing up information taken from different sources on the Web (Web pages, social networks, RSS feeds) and then publicly sharing these with other users who may be inter ested in gathering the same information. Situational con tributors may be defined as people that start to contribute to the mashup application when a specific event of interest occurs. For example, during a disaster people might want to publish pictures or information about the state of the damages originated by the phenomena. This is a category to take into account when designing mashup systems deal ing with emergency situations.

In Table 1, we present a categorization of the literature and systems previously reviewed according to the four dimensions described above.

By looking at Table 1 we can see that systems have different purposes but all share similar characteristics. In the next section, we present eStoryS and how the four dimensions have been taken into account when designing its functionalities such as georeferenced information (spatial), time intervals (temporal), collaborative filtering (collaborative) and storyboards editing and publishing (situational). 
Yahoo! Research Berkeley presents

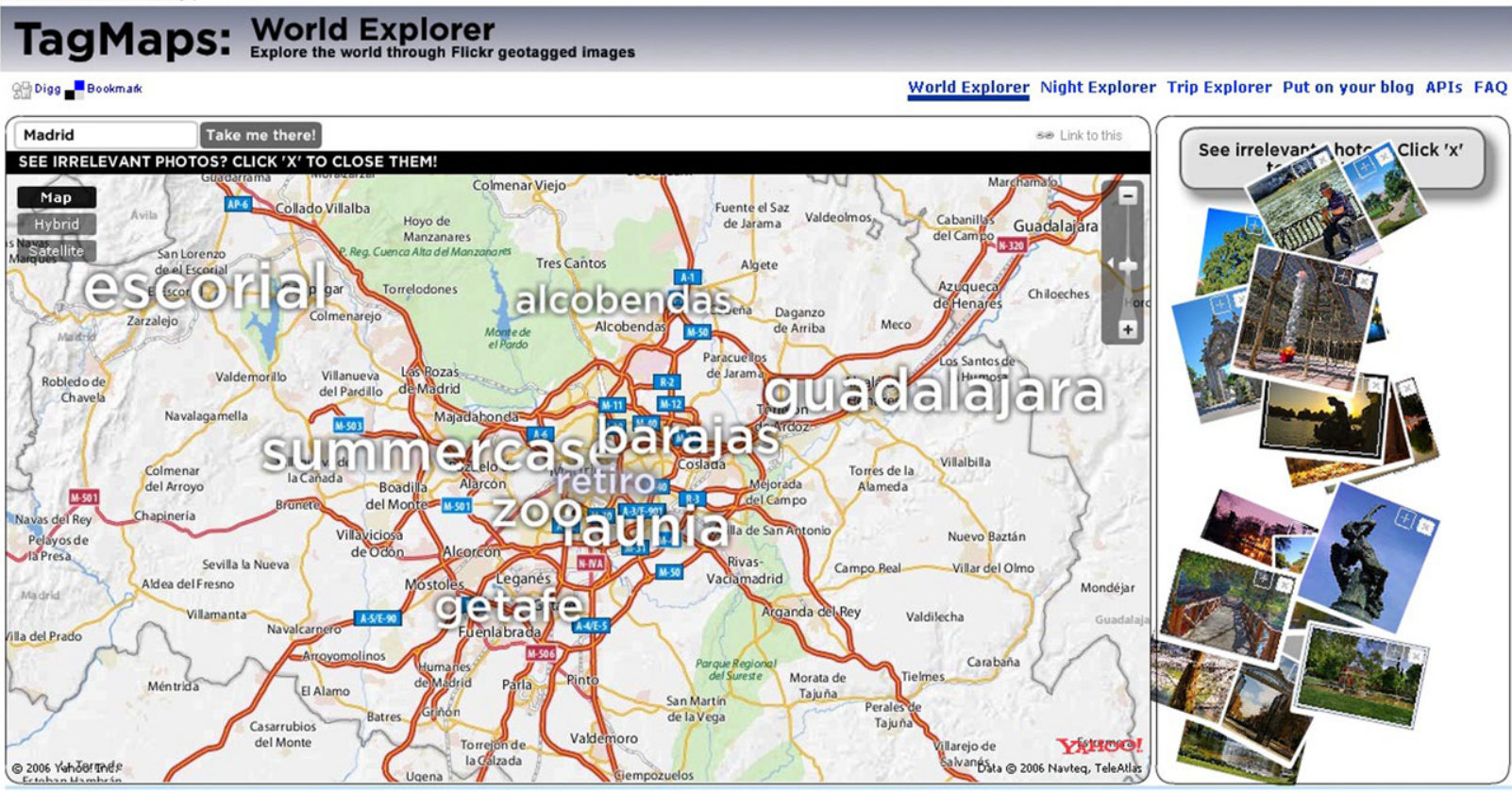

Quick links to fun places: Berlin Boston California DC Disneyland Egypt India Las Vegas London Maui NYC Paris Rome Switzerland Sydney Tokyo

Copyright $\odot 2006$ Yahool Inc. All tights resenved. Privacy Polieg I Copyright Notice I Ietms of Senvice I Contact Us

Fig. 3. Yahoo!'s World Explorer: the user selects a tag to visualize photos for that specific area.

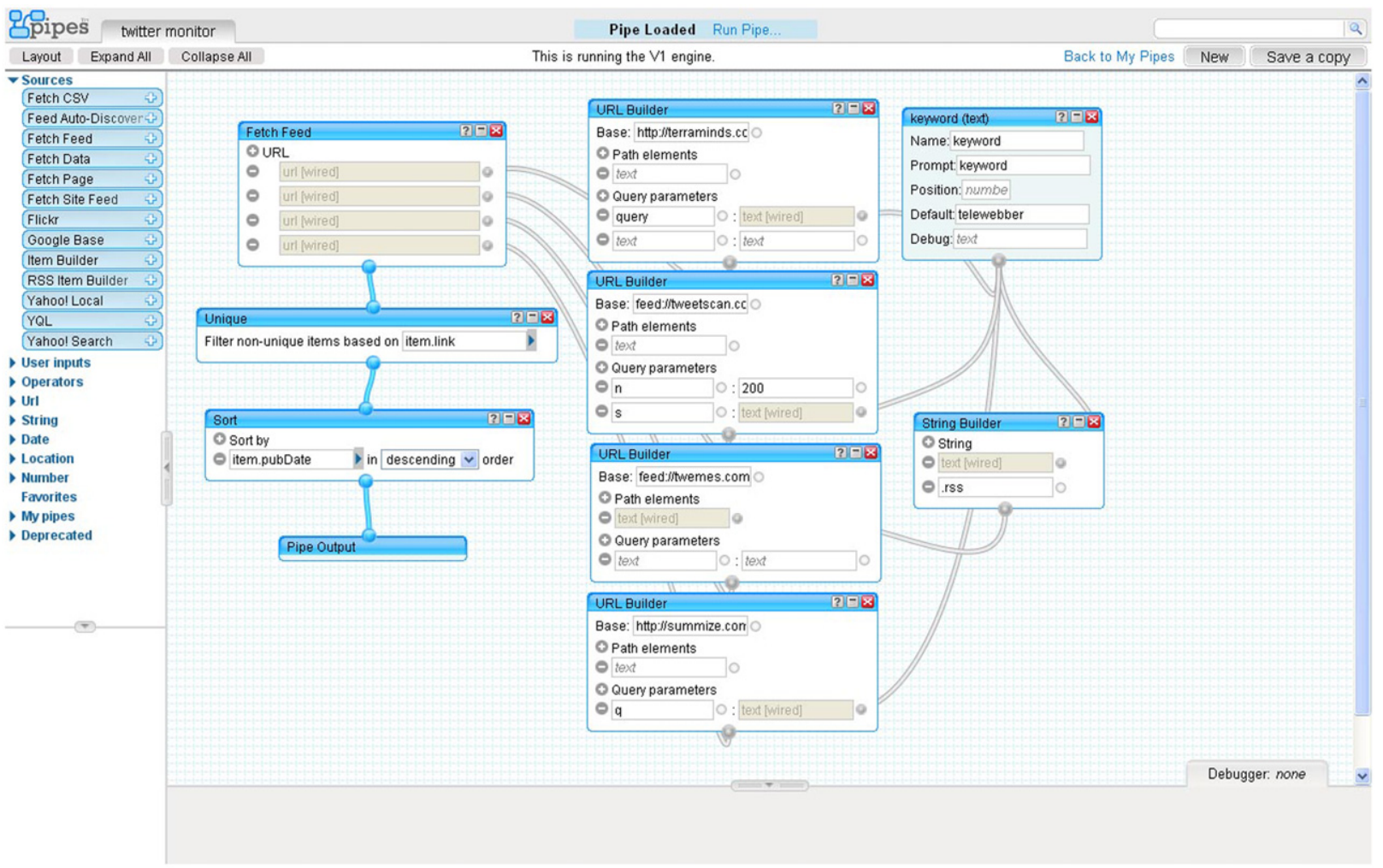

Fig. 4. Yahoo! Pipes. 
Table 1

Classification of mashup applications according to the four identified dimensions. (*) Georeferenced items are ranked by the system following their temporal order, starting from the most recent one. $\left(^{* *}\right)$ The relevancy of an image with respect to others is calculated by the system proprietary algorithm. The system ranks the images to visualize by means of this relevance measure.

\begin{tabular}{llll}
\hline System name & Spatial & Temporal & Collaborative \\
\hline Flickr Maps & Georeferenced images & Most recent $\left({ }^{*}\right)$ & Most relevant $\left({ }^{* *}\right)$ \\
Panoramio & Georeferenced images & Most recent $\left({ }^{*}\right)$ & Most relevant $\left({ }^{* *}\right)$ \\
ChicagoCrime.org & Georeferenced news & Most recent $\left({ }^{*}\right)$ & NO \\
Earthquakes mashups ${ }^{\mathrm{a}}$ & Georeferenced news & Most recent $\left({ }^{*}\right)$ & NO \\
Ushahidi & Georeferenced reports & Nemporal intervals & NO \\
Sahana & Georeferenced news & Specific date & NO \\
HurricaneArchive.org & Georeferenced reports & Specific event & NO \\
\hline
\end{tabular}

a http://earthquakes.googlemashups.com/ and http://earthquakes.tafoni.net/

b Multimedia content.

${ }^{\mathrm{c}}$ Hurricanes Katrina and Rita.

\section{3. eStoryS: emergency storyboard system}

We describe here our system. ${ }^{20}$ that exploits geo graphic location tags on digital photographs.

The rise of photo sharing services like Flickr and, of course, the proliferation of image capture devices have resulted in huge online picture databases contributed by the users. Thanks to the availability of an API, developers (skilled as well as occasional) can easily access such databases and build new applications relying on the stored information. Along with images, associated meta data can be retrieved. These metadata are valuable in understanding photo content and consist of textual infor mation such as keywords describing the picture (tags), the identity of who took the shot and the date when the picture was taken. Location information, such as latitude and longitude, identifying the geographical position where the picture was taken, can be available [31] too.

We designed our system by considering the four dimensions described in Section 2: spatial (georeferenced pictures: latitude and longitude), temporal (date and temporal intervals), social (recommendation and colla borative filtering) and situational (storyboard). Referring to Table 1 presented in the preceding Section 2.2.1 we highlighted characteristics and limitations of explored systems existing in the literature and on the web. The explored systems have limitations in the sense that those considering the collaborative dimension generally do not include features for explicitly managing the situational dimension (e.g. Flickrmaps and Panoramio). On the other hand, systems supporting situational dimension do not provide any form of collaborative filtering thus inhibit strong collaboration when it comes to publishing information instead of visualizing them (e.g. Ushahidi, ChicagoCrime). Diversely from these systems, eStoryS includes all the four dimensions in this design and the result is an integrated and general system for supporting back channels communications over georeferenced images on the web.

Our mashup application employs Flickr's API to retrieve pictures from its database and make use of

\footnotetext{
${ }^{20}$ http://estorys.spain.sc Login as guest (password: guest).
}

location metadata to accurately place such images on a map, exploiting Google Maps API.

In Fig. 5, the system interface is shown. Users can search for a geographic area entering any combination of address, city, state or zip code. Subsequently, the system retrieves all the georeferenced photos taken within the selected area. Finally, the retrieved pictures are placed on the map according to their location (spatial dimension). Up to five zoom levels are supported, from a country view (lower) to a street view (higher). Zoom levels also affect images visualization on the map. While, at lower levels, images are clustered into placemarks (according to their geographic distance), images thumbnails are placed directly on the map at the higher level.

Users can browse for photos by selecting (a) the associated place holder on the map or (b) the image thumbnail, placed in a ranked list present in a panel. The use of thumbnails appears to be effective in user interfaces for the visualization of digital images [1], because of their capacity to gather a lot of information in a small space. Furthermore, a vertical scroll bar allows users to access thumbnails not visible in the panel.

Double clicking either a place holder or a thumbnail provides a full view of the image (see Fig. 6). A single mouse click, instead, enables users to visualize further information about the images, like the associated tags, the photo's title, its owner, the date and the geographic position.

Pictures are retrieved also considering the temporal dimension, in conjunction with the spatial one. The system interface enables users to select temporal intervals and subsequently retrieve the photos with a shot date within the given range. Through our collaborative mapping mashup users can create and share their own pictorial content, rather than simply browsing and visualizing geolocated images.

Lastly, registered users' information as well as the history of their interactions with the system are stored in a database on the server. Such information turns out particularly useful to analyse the users' behaviour and to design tools embracing the users' collaboration. As a result, we have developed a naive recommendation sys tem [22] as a means to filter and rank the retrieved pictures for exploiting the social dimension. In emergency situations, involved people are under pressure to absorb 


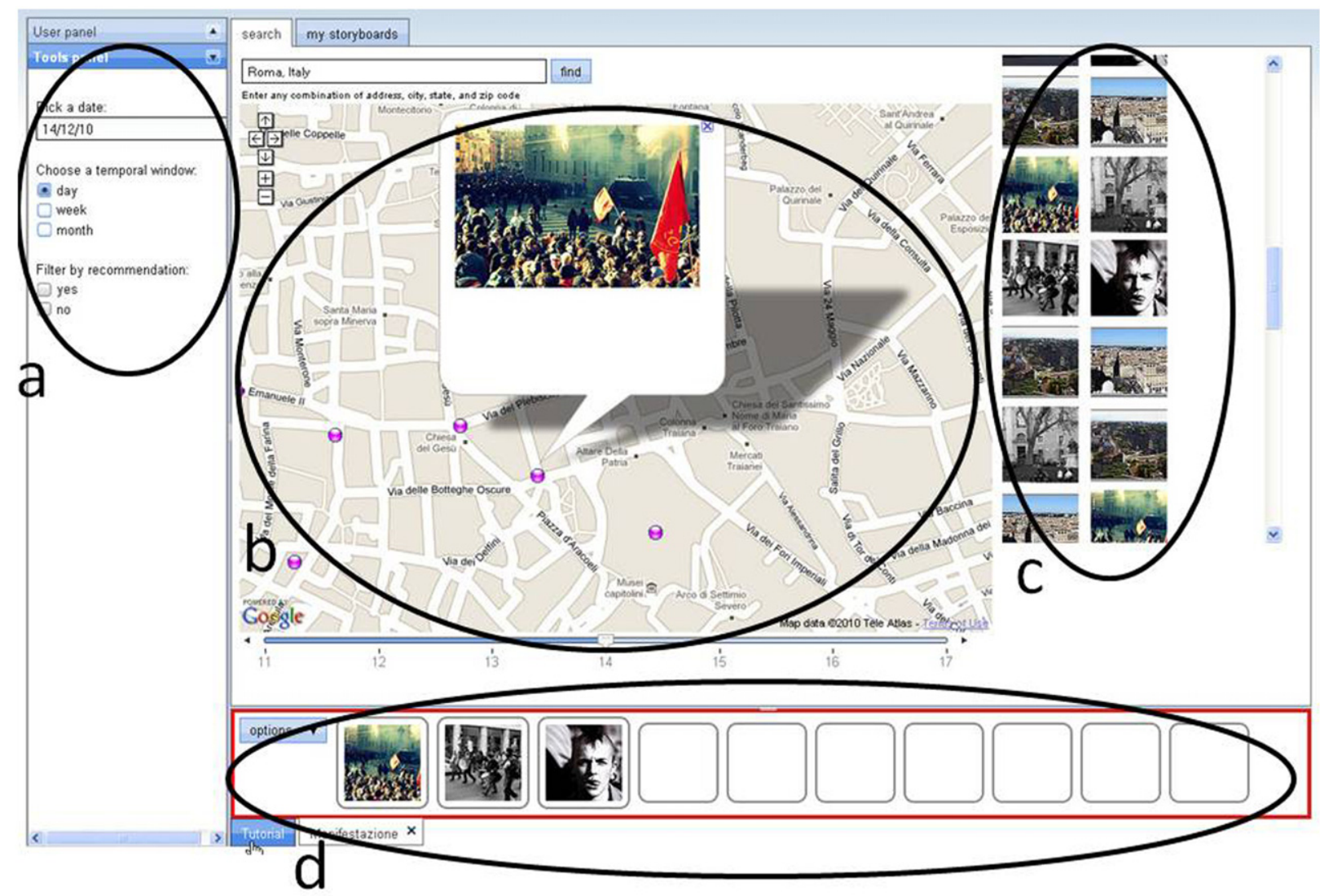

Fig. 5. The user interface of our prototype application. (a) Temporal and filter settings; (b) digital map panel; (c) ranked list of retrieved images; (d) storyboard authoring panel.

information rapidly, to judge their relevance and relia bility and to make effective decisions [7]. For these reasons, systems supporting disaster management must help users in facing this information overload, providing ways to obtain available information quickly and possibly with minimum effort. We describe in Section 3.4 how implicit users collaboration (through collaborative filter ing) can be successfully exploited to satisfy these needs. Finally, we provided a Storyboard Authoring mode, in which storyboards of selected images can be edited. Storyboards are graphic organizers, such as a series of illustrations or images displayed in sequence. Although the storyboarding process has its roots in the film indus try, the term storyboard has been used recently in the fields of web and software development to present and describe interactive events, particularly on user inter faces, electronic pages and presentation screens. The use of storyboards according to the situational dimension help situational contributors (people publishing photos during a specific event or for a specific purpose like an emer gency) to group photos and publish sequences of events on the system.

\subsection{System development}

Two main web services have been developed. The first service is responsible for making calls to the Google Maps'
GClientGeocoder class (provided by Google's API) to communicate directly with Google servers, in order to map the address, as entered by the user, to its geographi cal coordinates. Such coordinates are employed by the second service, that queries Flickr to retrieve the required information, according to the spatial temporal con straints. Data are exchanged by means of the Javascript Object Notation (JSON), a lightweight standard format that is easy to read and write for humans, as well as it is easy for machines to parse and generate. On the client side, information is extracted by parsing the retrieved JSON archives.

We have made extensive use of the AJAX web devel opment technique to build the system interface as well as for visualizing content. Several AJAX libraries, such as the Dojotoolkit, ${ }^{21}$ provide a wide range of pre built UI (User Interface) components and effects, in order to provide a fast development of rich internet applications. As an example, our approach to manipulate images to place into storyboards employs drag and drop. This technique results fast and easy to learn for users to perform tasks, having the advantage of thoughtfully clumping together two operands (the object to drag and the drop location) into a single action [6].

\footnotetext{
${ }^{21} \mathrm{http}$ //dojotoolkit.org
} 


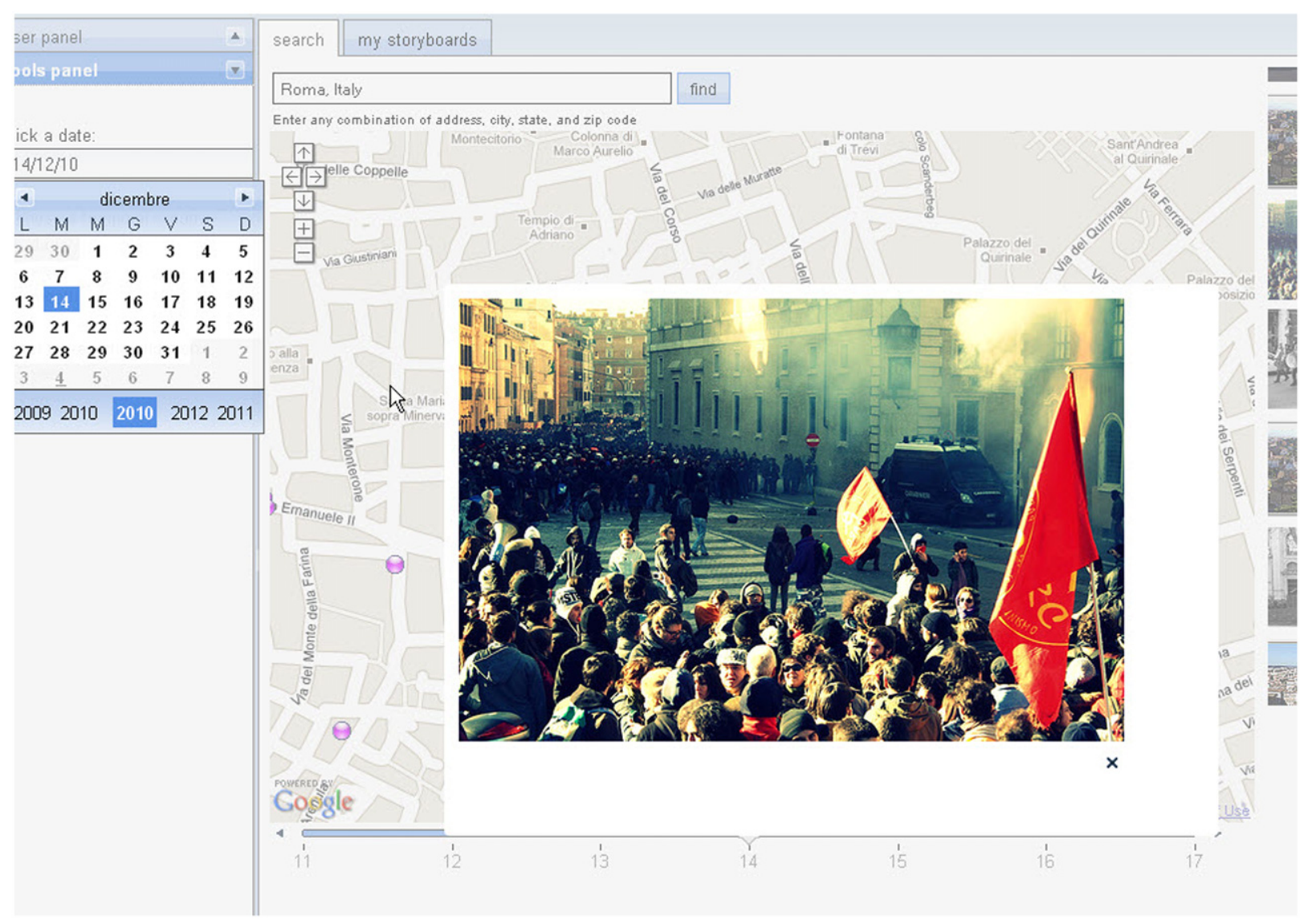

Fig. 6. Full-view of a selected thumbnail. This shot was taken at the Atocha station in Madrid on 13 March 2004.

\subsection{Time based retrieval}

We have also implemented three basic components (see Fig. 7) in order to specify constraints on the temporal properties: (a) the calendar, (b) the temporal interval box and (c) the timeline (a temporal slider [28]). Obviously, these components allow users to constrain their query by time.

The calendar component consents to select the date of photos to retrieve. For example, if we are interested in obtaining pictures of the $11 \mathrm{M}$ terrorist attack in Madrid, we have to enter Atocha, Madrid, Spain in the search box and select the date of 11 March 2004 from the calendar. The temporal interval box allows to define a timespan of one day, one week or one month. Consequently, the system will retrieve photos being shot within the selected range, starting from the chosen date in the calendar (see Fig. 6). The timeline slider is a widget, displayed in a horizontal fashion, with which a user may shift the temporal window by moving an indicator. Fig. 7c shows the resulting timeline slider for a temporal interval of one day. Users can retrieve and visualize photos of the days immediately before or after the selected one by simply clicking with the mouse on that day or, of course, drag ging the indicator on it. The same holds for weekly and monthly time spans.
These components result really helpful to make the system practical, avoiding that a query returns a huge number of items. In fact, they can be thought, in conjunc tion with zooming on a particular region, as a primary information filtering tool. As an example, users can reduce the amount of retrieved data by simply narrowing down on a geographical area and, at the same time, decrease the temporal window. Moreover, the presence of widgets for defining temporal constraints, helps users in refining their queries. In this way, they can immediately retrieve the information they need, avoiding to search in large messy collections of images. During emergencies it is crucial to quickly obtain information on the disaster area, in order to organize relief operations. However, it is equally important to have a clear view of the area before and, immediately after the disaster occurs, report on damages estimation as well as monitor (and provide updates on) post disaster operations.

\subsection{The storyboard tool}

Our system also provides a tool to quickly generate storyboards exploiting drag and drop of selected images (Fig. 8). Therefore, in order to create a storyboard, a user can select pictures from the list of retrieved images (the panel on the right in the system GUI, see Figs. 5c and 8a) 


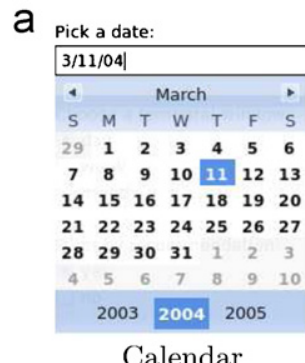

Calendar b

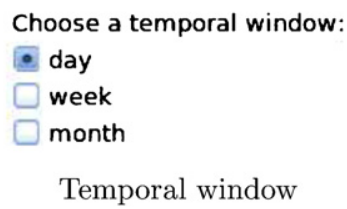

C

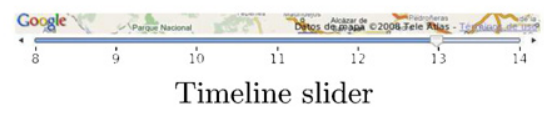

Fig. 7. UI components to specify temporal constraints on queries. (a) Calendar. (b) Temporal window. (c) Timeline slider.

and drag such images directly into the storyboard panel (a tabbed pane identified by the storyboard's name, see Figs. $5 \mathrm{~d}$ and $8 \mathrm{~b}$ ). A menu gives the possibility to save the storyboard, as well as to edit its attributes (Fig. 8b). Asso ciated with each storyboard there is a colour, indicating the emergency intensity rating: green for low, yellow for moder ate and red for high. Suppose a user is interested in building a storyboard on an emergency situation. Depending on what the storyboard will be about, she can assign: (a) a red colour, in case the storyboard contains photoshots taken during such emergency, (b) a yellow colour, for events occurred immediately after the crisis or, finally, (c) a green colour, for images referring to the recovery [14] phase (i.e. damaged building or infrastructure).

Once generated, a special marker representing the storyboard is placed on the map, according to its spatial features and visual metaphors described in [3] and shown in Fig. 8c. User generated storyboards are stored in a database, containing information like: the owner, the URLs of related photos and the spatial temporal data. The storyboard's geographic position is estimated as the centroid (or geographical centre) of the region detected by the coordinates of its photos. A time span, connected to each storyboard, represents its time duration and corre sponds to the previously selected temporal interval.

Storyboards can be viewed by all other users and filtered depending on the kind of emergency level (green, yellow and red). Moreover, the use of storyboards can stimulate and help situational contributors since we think that when a disaster occurs many citizens could refer to such a system for the first time to publish storyboards. The storyboarding process addresses both common people as well as members of governmental agencies. As an example, citizens can build storyboards to report, to relatives or friends, on the status of their personal property. Meanwhile, professional officers may use this tool for damages estimation, highlighting a region before, during and after a disaster occurs. These are exactly a kind of phenomena we identified in Section 2 for which we considered the situational dimension.

\subsection{Ranking through recommendation}

Recommendation algorithms are the best known for their use on e commerce systems, where information about a customer's interests is employed to generate a list of recom mended items. Such information includes, other than the items that customers purchase, items viewed, demographic data, user's interests and preferences. There are three main approaches to handle the recommendation problem: tradi tional collaborative filtering, cluster models, and search based methods [22]. In traditional collaborative filtering, recommendations from similar customers' items are selected using various methods. A common technique is to rank each item according to how many similar customers purchased it. We employ here a similar approach. Users are viewed as a $N$ dimensional vector of queries, where $N$ represents the number of different queries performed by the user. Every query is represented as an $M$ dimensional vector, where $M$ is the number of retrieved images. A Boolean value is associated to such images, and it is: true if the photo was viewed by the user (double clicking on the place holder or the thumbnail), false otherwise. The system ranks images according to how many different users have double clicked on it. The ranking is computed on the information contained on the correspond ing cell of the vector of all the users which performed a given query. We assume here that during, or immediately after, an emergency the most viewed images for a given area are probably the most relevant ones, with respect to the specific emergency (e.g. photos of damaged buildings, firefighters rescuing people, etc.).

At this stage we employed recommendations only for ranking purposes. In the future work, we plan to further investigate the use of such techniques in collaborative systems for emergency management, as well as to employ different recommendation algorithms.

\section{System evaluation}

\subsection{Analytic evaluation through a scenario}

We evaluated our system by comparing it with other analogue systems publicly available. These systems have been carefully selected among mashup applications explored in Section 2. We selected FlickrMaps and Panor amio considering them as the only ones comparable to our system. Even if Ushahidi might seem similar too it presents some evident limitations that might have affected our analytic evaluation. In particular, map based mashups developed using the Ushahidi engine are geo graphically limited to a specific scenario. eStorys provides an interface for searching and selecting geographic areas among the world, as FlickrMaps and Panoramio do, while 

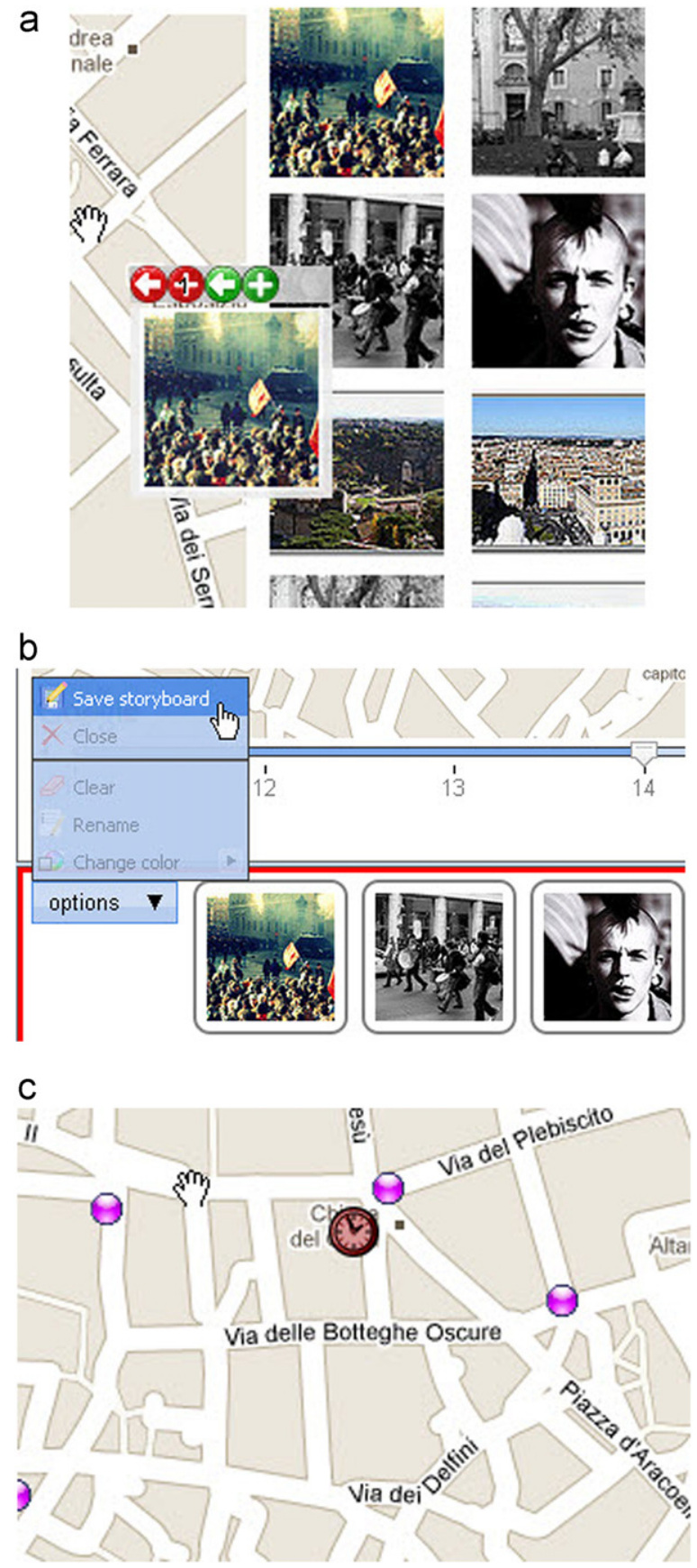

Fig. 8. The storyboarding process. (a) Drag-and-drop. (b) Save. (c) Placing a marker on the map.

Ushahidi is restricted to specific areas (selected by the designer depending on the specific event); thus our system is not directly comparable with mashups gener ated by Ushahidi that at a first look might seem similar to eStorys.

Following the analytical evaluation technique [26] we designed two scenarios that represent typical situations where our system, and this kind of mashup systems could be of greatly helpful.
In 2005 Hurricane Katrina was one of the deadliest in the history of the United States. Among recorded Atlantic hurricanes, it was the sixth strongest ever. Hurricane Katrina formed over the Bahamas on August 23, 2005, and crossed southern Florida, causing deaths, flooding and destruction along the coast of Gulf of Mexico from central Florida to Texas. The most shattering loss of lives and property damage occurred in New Orleans, Louisiana, which flooded due to the floodbank system failure. Let us imagine that today is Wednesday, 31 August 2005. One of your best friends lives with her/his family in Loyola Avenue, New Orleans. You are worried about her/him because he/she does not answer the phone and stopped updating her/his blog. You are interested in obtaining information (photos in our case) on the affected area, to be aware of the extent of the damage and, with luck, to know something about your friends' health. How can you take advantage of current mapping services to accomplish this task?

Flickr Maps (Fig. 9) offers an interface to search for arbitrary areas in the world, using a map to display photo items, like our system does. Nevertheless, analysing the Yahoo system, we conclude that it is unsuitable for the presented scenario. In order to find representative pic tures, users have to look over a large number of images, by using a slideshow widget provided by the system interface. There are currently about 73000 georeferenced photos for the Loyola Avenue's area in the Flickr database, and only a subset of about 20 images at a time is presented to the users. Photos are ranked depending on their interestingness in the Flickr community, or their upload time on the website. Moreover, users cannot retrieve pictures exploiting the temporal dimension in conjunction with the spatial one; for example, by select ing the date when photoshots were taken, or even within a temporal interval.

Panoramio (Fig. 10), the mapping service offered by Google, incurs in the same limitations as Flickr Maps, if employed in the emergency management domain. Panor amio provides the users with an interface where a subset of retrieved photos are visualized in a panel on the left and image thumbnails are placed directly on a map within the main panel. In addition, this system does not implement an interface exploiting the temporal dimen sion for querying its images database. Pictures are ranked only by popularity or upload time. Like in the Yahoo system, in order to identify representative images, users have to scroll over the subsets of retrieved pictures. To summarize, it is clear that searching images of a particular event, when using these two systems, can be really a hard and time consuming chore.

Conversely, with our system (see Fig. 11), interested people can easily acquire useful information. Using the calendar widget (Fig. 7a) and the temporal window widget (Fig. 7b), users can exploit the temporal metadata in order to retrieve only the subset of photoshots taken in a given temporal interval, depending on the selected date. Users displace over contiguous temporal intervals by means of the timeline slider (see Fig. 7c). Finally, the entire set of retrieved images is visualized in an assigned panel (as well as on the map); such pictures are ranked through our 


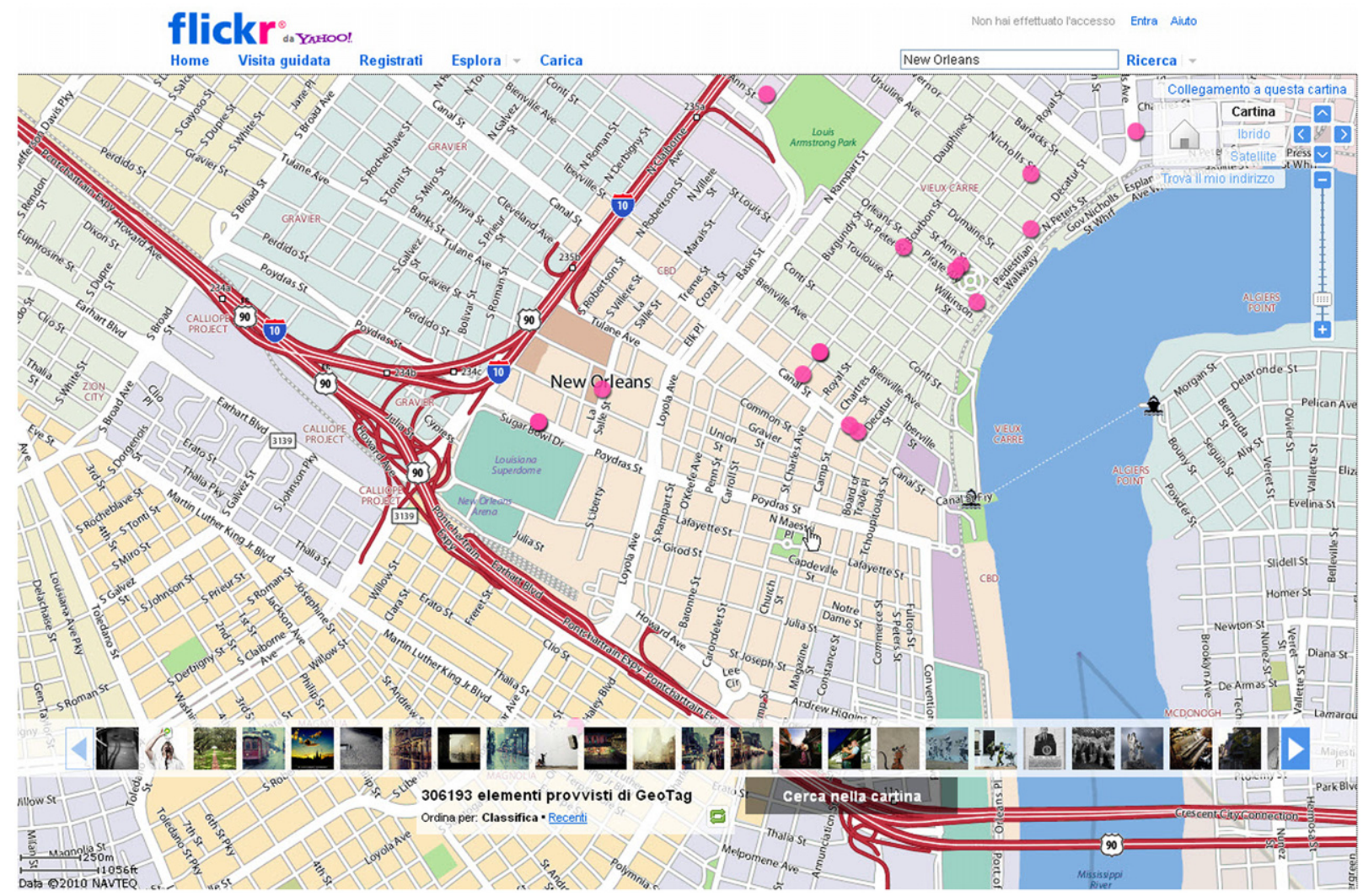

Fig. 9. Loyola Avenue, New Orleans, Louisiana on Flickr Maps.

recommendation system, taking advantage of the users' collaboration. Taking into account the presented scenario, in order to find related photos to her/his friend's safety, a user has to select the date in which the Hurricane Katrina made its landfall in New Orleans (Monday, August 29th, 2005), to set the temporal interval of a week and, of course, to type the address Loyola Avenue, New Orleans, Louisiana, US in the search box. As a result, the system will place retrieved photos on the map and simply after selecting photoshots located in Loyola Avenue, the user can determine the situation (damaged building, citizen's safety, etc.) arisen in that place.

Now, assume you are a member of the Civil Defense (a professional working in the emergency field), having to deal with this catastrophic event. One month after the crisis you have to report on the passage of the hurricane, damages and recovery operations, certificating it with photos. You have to choose a set of photos to build a sequence of images, describing the situation in New Orleans, before the hurricane occurs, during the disaster and immediately after.

We have just highlighted how difficult it can be to retrieve pictures of an event employing Flickr Maps or Panoramio. Moreover, these two systems do not provide any tool to build temporal sequences of images. Due to this fact, in order to accomplish this particular task, a user should manually build the sequence, resulting in a bur densome activity. She should provide, for example, a directory structure on their personal computer (based on the pictures date), where selected images were stored. Nevertheless she cannot acquire the date in which a picture was taken until she explicit selects it, and this temporal metadata cannot be stored along with the image. To this end, she could create a directory (with a name depending on how she wants to title her/his sequence) and then add a sub directory for each of the selected images, named with the photoshots date.

With eStorys, the process of generating sequences of images is fast and simple, thanks to the presence of the storyboard tool (see Fig. 12). Building a storyboard of images only consists of (a) selecting the storyboard active time (one day, one week or one month) (b) defining the storyboard severity rating (red, yellow or green) (c) assign ing a title to the storyboard and finally (d) populating the storyboard by dragging selected photos. The widgets pro vided by the eStorys interface turn out to be really helpful. A user is always conscious of the pictures date due to the presence of the calendar widget. The temporal window widget allows a user to select tighter or wider temporal intervals and the timeline slider to quickly shift between them.

In this first step in the development of the mashup system for back channels communication during emergen cies we were mainly interested in the use of images for describing the status of the disaster or to contribute to grassroots journalism and for this reason we restrict to the image media. Moreover, georeferenced images are very 


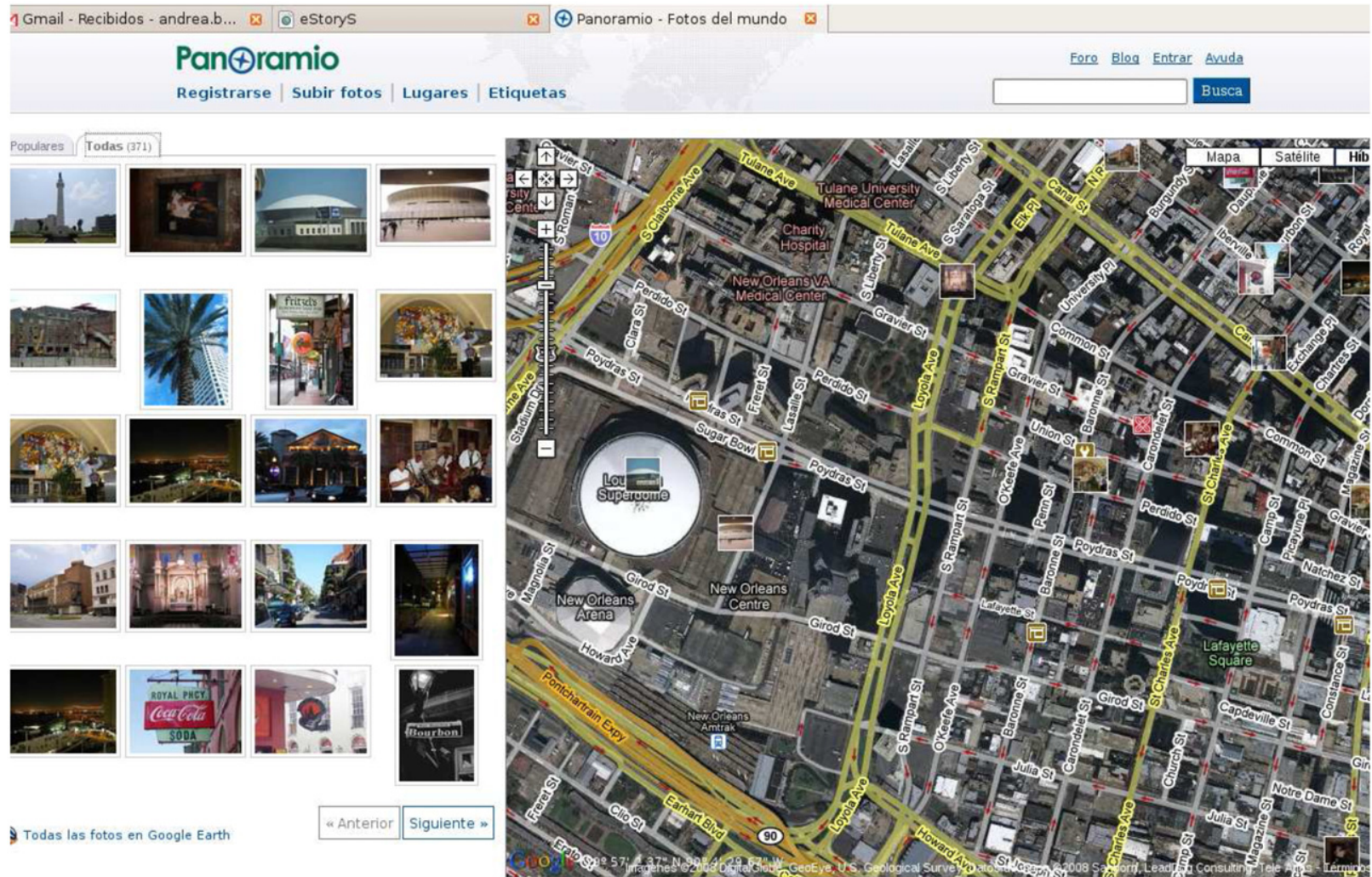

Eopyright 2007 Google.com. Todos los derechos reservados. | Las fotos están bajo el copyright de su propietario. | Blog | Ayuda y soporte

Fig. 10. Loyola Avenue, New Orleans, Louisiana on Panoramio.

important for rescue planning or damages evaluation during a disaster. Nevertheless, our background study and future works points toward the integration of more media.

\subsection{Heuristic evaluation}

To evaluate the usability of our mashup application, we conducted a heuristic evaluation, according to the discount usability approach [24]. Our expert reviewers examined the interface design to determine its compli ance with a short list of usability principles (called heuristics). The 12 expert reviewers were carefully selected among a group of graduate students of the Computer Science Department at University Carlos III of Madrid, Spain. They all attended an advanced seminar on $\mathrm{HCl}$ and usability and thus could be considered quite expert in applying usability guidelines. The heuristics used for conducting our experiment are general rules that intent to describe common properties of usable interfaces. Individual evaluators performed the evalua tions, each inspecting the interface alone. We demanded not only to say that they do not like something, but also to explain why they do not like it, with reference to the heuristics. We exploited here, as heuristics, the eight human factors considerations, identified by Lin et al. in [16]. These factors are compatibility, consistency, flexibility, learnability, minimal action, minimal memory load, perceptual limitation and user guidance. Since our application addresses both common people and members of governmental agencies, it was not strictly required for the evaluators to be expert on the domain (emergency management). The evaluators received a 10 min explanation of the system and its main function alities. As we were also interested in assessing the efficacy of the online tutorial of the system, no observers attended the evaluation sessions. In case of problems or doubt, experimenters can only receive hints looking at the tutorial. Therefore, during the sessions, the experts exam ined the interface several times and reported a list of usability problems in the interface, as well as positive aspects, with reference to the previously defined heuris tics. For each heuristic, we have identified subcategories, in order to categorize evaluators' findings. As an example, the compatibility heuristic consists of the four subcate gories of Common Vocabulary, Keywords, Icons \& Com mands and Browsers.

Fig. 13 shows the results of our evaluation with respect to each heuristic. The $83 \%$ of the evaluators reported on serious compatibility problems regarding the keywords used to identify functionalities peculiar to the eStorys application, like the temporal window or filter by recommendations. In fact, these labels may be unfamiliar to the user, which could 


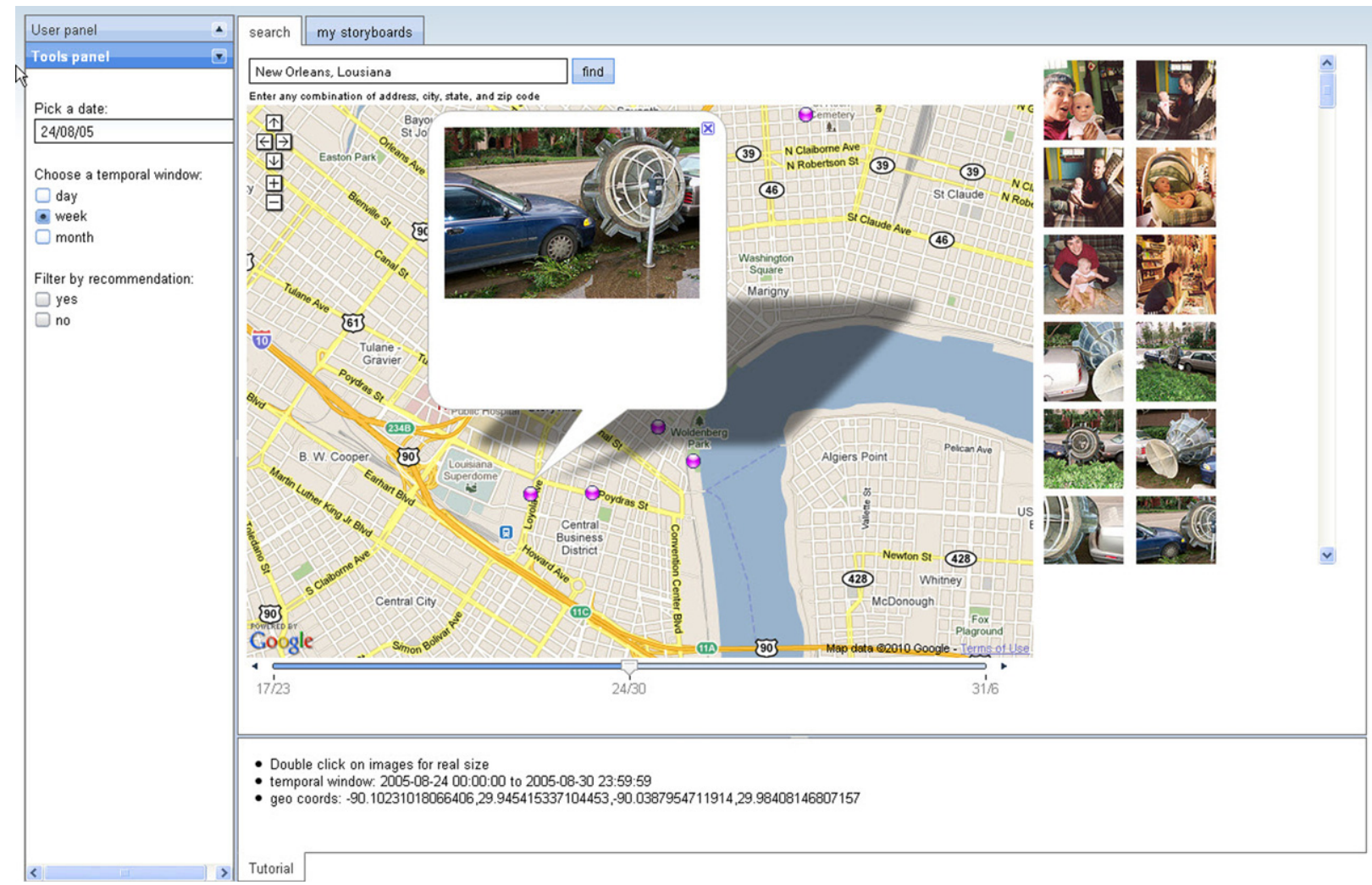

Fig. 11. Loyola Avenue, New Orleans, Louisiana on eStorys.

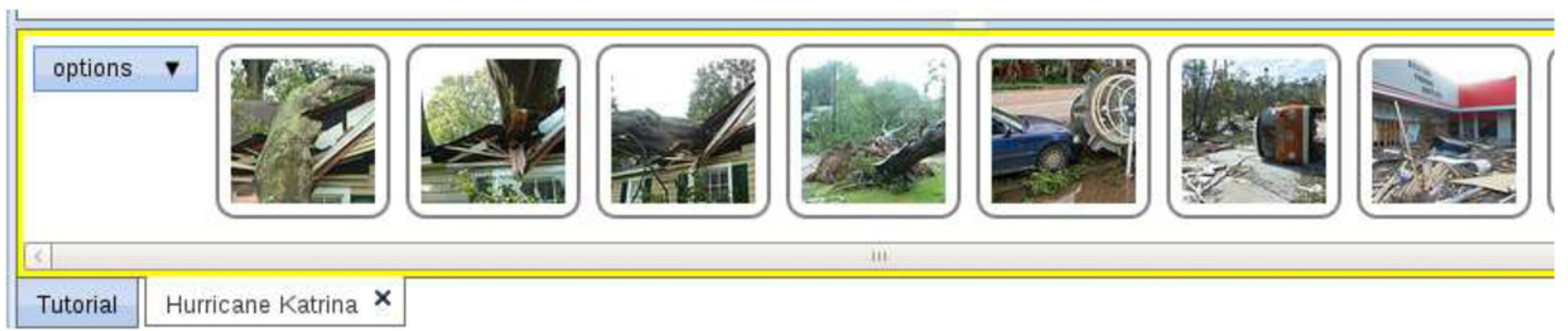

Fig. 12. A storyboard including damages with different perspectives built after the hurricane Katrine.

not understand well the function of the specific UI compo nent. As reported by one of the evaluators:

I found the labels used to identify functionalities peculiar to the system very confusing. I cannot under stood what the temporal window refers to, before I started interacting with the system.

On the other hand, the $83 \%$ of the evaluators found consistent with the use of the three colours (red, yellow and green) to identify the severity rating of an emergency storyboard. In fact, our system employs the same colour code, as defined at the time of storyboard creation, to distinguish UI elements related to the storyboard; clear examples are the border framing the storyboard authoring panel and the icon representing the storyboard on the map. As one of the evaluators explained in her report:

I found consistent the use of the three colours: red meaning emergency, yellow for alarm and green for a normal situation.

Overall, it resulted that the majority of usability flaws only concerns cosmetic aspects of the system interface that can be rapidly enhanced. The $42 \%$ of the evaluators also reported on the lack of a tool for uploading and sharing personal photoshots, apart from the images gath ered from Flickr. However, they positively assessed the adopted interaction techniques and system functionalities 


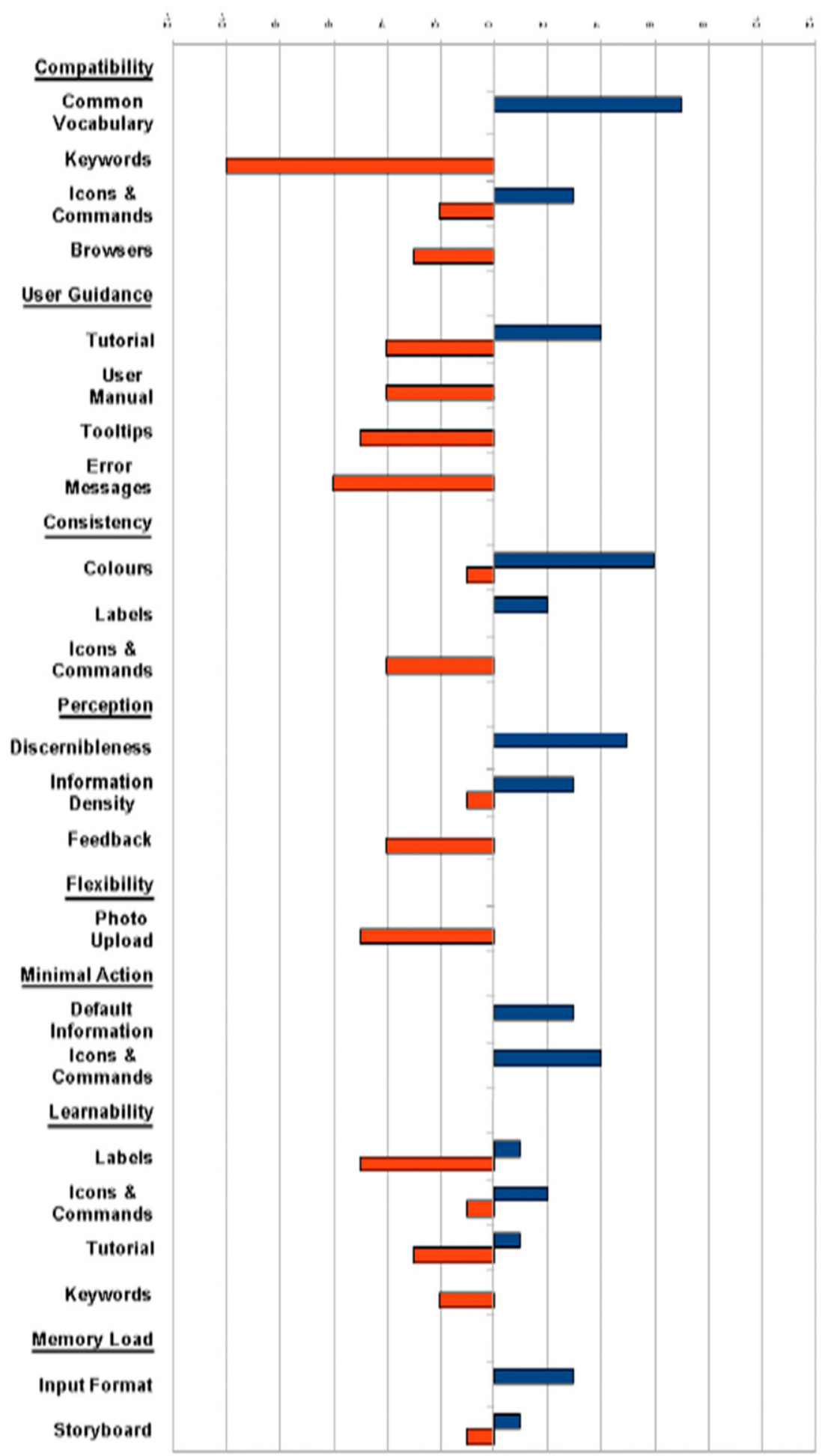

Fig. 13. Graphic of evaluators' findings with respect to the given heuristics. Dark bars correspond to positive evaluations, while light ones to negative. On the $x$-axis all the parameters evaluated for each category are presented. We exploited here, as heuristics, the eight human factors considerations, identified by Lin et al. in [16]. 
for handling temporal and georeferenced online photo collections. As one of the evaluators stated:

I can create spatio/temporal storyboards in an efficient and intuitive way. I think the overall usability of the interface is satisfactory, with respect to the system objectives.

\subsection{Experimental evaluation}

We conducted an experiment with 34 participants, which were introduced to the system by using an online tutorial we prepared. Participants were asked to perform three tasks of incremental difficulty and to fill a post task questionnaire.

The overall duration of the experiment was around $2 \mathrm{~h}$. The first 15 min were spent to give a brief explanation of the system and an introduction to the purposes of the experiment. The participants spent the rest of their time in using the tutorial, completing the tasks and answering the questionnaire.

The three tasks we asked to complete were related to the specific use of eStoryS in the domain of emergencies; the first one was generic, the second one required the use of the timeline to solve the task more efficiently; while the third and the last one required the use of the story board tool to collect information about the scenario (see Appendix A for details and questionnaire design informa tion). We will refer here to questions in the questionnaire (see Appendix B) by using a short sentence for the topic and the question number, like for instance Q1 indicating the question number one. From the first three questions (Q1, Q2, Q3) we extracted information about the back ground of the users. It resulted that $27 \%$ of the

Table 2

Statistics about participants.

\begin{tabular}{ll}
\hline Age range & $18-34(100 \%), 18-24(70 \%)$ \\
Sex & Male (65\%), female (35\%) \\
Education & $\begin{array}{l}\text { Computer Science (18\%), Technical } \\
\text { Engineering Computer Managements } \\
(44 \%), \text { Computer Engineering (38\%) }\end{array}$ \\
Job title & $\begin{array}{l}\text { Student (76\%), developer (12\%), other } \\
(12 \%)\end{array}$ \\
$\begin{array}{l}\text { Use of computer for } \\
\text { main activity }\end{array}$ & \\
\hline
\end{tabular}

participants have already used Flickr while $73 \%$ have not used it before. From Q2, we noted that $41 \%$ of the participants had an idea of what a mashup is and have already used it, while 59\% was not aware of this term. Concerning the use of web mapping applications (Q3) 61\% of the answers were between 0 and 1 , which means never used a web mapping or used only one kind of web maps. We must point out that the category with the higher frequency, 35\%, selected one application (almost coincid ing with Google Maps). In Table 2, we present the statistics about our participants (questions in the users' profile section of the questionnaire). They were in the age range of 18 34, with 70\% of the population in the 1824 range. The age range is the one expected by the people most frequently using (and will use in the future) social and photo sharing applications on the web.

Fig. 14 shows the results for the first part of the questionnaire (from Q4 to Q12). A Likert scale of five values [21] was used in our questionnaire: strongly agree (1), agree (2), neutral (3), disagree (4) and strongly disagree (5). We grouped answers to question from Q4 to Q12, because they represented a general evaluation of the system. The graph in Fig. 14 represents the percentage of positive answers ( 1 and 2 in the Likert scale), neutral answers (3), and the percentage of negative answers ( 4 and 5 in the Likert scale). As we can see the general score is positive. Especially Q4 and Q5 (concerning system interface and presentation of information) appear clearly positive. The tutorial was helpful, as proved by a $68 \%$ of positive answers. This implies a good understanding of the system and can affect the good results of Q4 and Q5. Only Q6 (unexpected behaviour of the system) is clearly negative. This can be due to the fact that elements of the interface were sometimes expected to perform different actions depending on the type of browser used for the experiment, leading to an unexpected behaviour of the interface components. We believe that results of Q7 and Q8 (system functionalities) were also influenced by this unexpected behaviour.

The average values of scores over questions from Q4 to Q12 are presented in Table 3. Table 3 helps us in under standing the magnitude of the positiveness or negative ness of the answers compared to the frequencies presented in Fig. 14. Summing up, the strongest point of our system are: the interface, the information clarity and the quality of the tutorial.

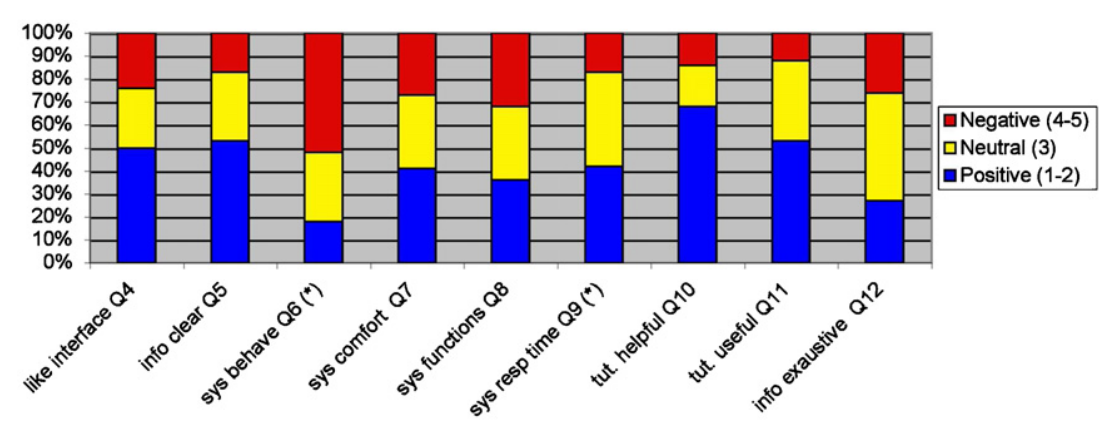

Fig. 14. The graph representing the percentage of positive against negative answers on questions from Q4 to Q12. Questions with (*) means that the question was posed in a negative but in the graph have been inverted to give an homogeneous overview. 
Fig. 15 shows the results obtained for the three tasks participants were asked to perform.

As we can see from the graph the overall judgement on the use of the system for completing the tasks was positive. We can highlight that it was particularly effec tive on task 3 (Q19, Q20, Q21) which was the most difficult one. We think that among the proposed tool, the storyboard could be of great help in such kind of tasks. We want to point out that 71\% of the answers to Q18 and $74 \%$ of the answers to Q21 were in the 12 range (strongly agree, agree). The only negative point here seemed to be on Q16, where participants judged as negative the com plexity for completing Task 2 , which could be due to an inherent complexity of the task we designed. In fact, participants judged with a positive result the time slider tool (used in Tasks 2 and 3) but might have happened that they did not find it easy to use for the selected task.

Table 4 displays the average values for questions from Q13 to Q21. By analysing the averages presented in Table 4 we can see that a general positive impression comes out from the completion of the three selected tasks.

In Fig. 16, we present results on questions from Q22 to $\mathrm{Q} 28$, related to the overall evaluation of the system with respect to the completed tasks.

As we can see from the graph in Fig. 16, the results are mainly positive. Specifically, question Q23 (easy of use of the system) scored clearly positively, with a few neutrals. This confirms that the users liked the interface and the

\section{Table 3}

Average scores on questions from Q4 to Q12. Questions with a $\left(^{*}\right)$ mean that the question was posed negatively but has been inverted in the graph for a homogeneous overview. In fact, for $\left(^{*}\right)$ questions: 4 and 5 were positive values, 3 neutral and 1 and 2 negative. Therefore only the mean of Q6 tends to a negative result.

\begin{tabular}{ll}
\hline Question & Average \\
\hline Q4 & 2.8 \\
Q5 & 2.6 \\
Q6 $\left(^{*}\right)$ & 2.5 \\
Q7 & 2.8 \\
Q8 & 2.9 \\
Q9 $\left(^{*}\right)$ & 3.2 \\
Q10 & 2.4 \\
Q11 & 2.5 \\
Q12 & 3 \\
\hline
\end{tabular}

presentation of the information and thus the overall users' experience with the system is good. Moreover, question Q25 (level of integration of system's functionalities) scored a good result, which was one of our aims. Since the system is the mashups, the level of integration of the different features is relevant for the users' experience with the system. If different functionalities are not well integrated, the system could present a heavy cognitive load for the user in trying to understand which different web systems have been mixed for generating the mashup application, leading to a non coherent interface and users' interaction.

In Table 5, we can see that the averages are in line with what expected from the frequency analysis presented in Fig. 16.

Summarizing all the results, we grouped the positive and negative findings in Table 6 . There is an evidence that users liked the interface, the information organization and the provided tutorial. Furthermore, the system resulted easy to use and functionalities appeared well integrated. This last characteristic is quite relevant since eStorys is the mashup application and thus it is an integration of different sources of information and systems (Google Maps, Flickr, and so on). On the other hand, the system resulted partially unstable when tested on different browsers (this can be due to the peculiarities of the technologies which are not completely standard when rendered in different browsers).

\section{Conclusions and future work}

In this paper we presented a mashup system for helping people and professionals to cope with emergencies.

Table 4

Average scores on questions from Q13 to Q21.

\begin{tabular}{ll}
\hline Question & Average \\
\hline Q13 & 2.1 \\
Q14 & 2.2 \\
Q15 & 2.4 \\
Q16 & 2.9 \\
Q17 & 2.6 \\
Q18 & 2.2 \\
Q19 & 2.3 \\
Q20 & 2.4 \\
Q21 & 2.2 \\
\hline
\end{tabular}

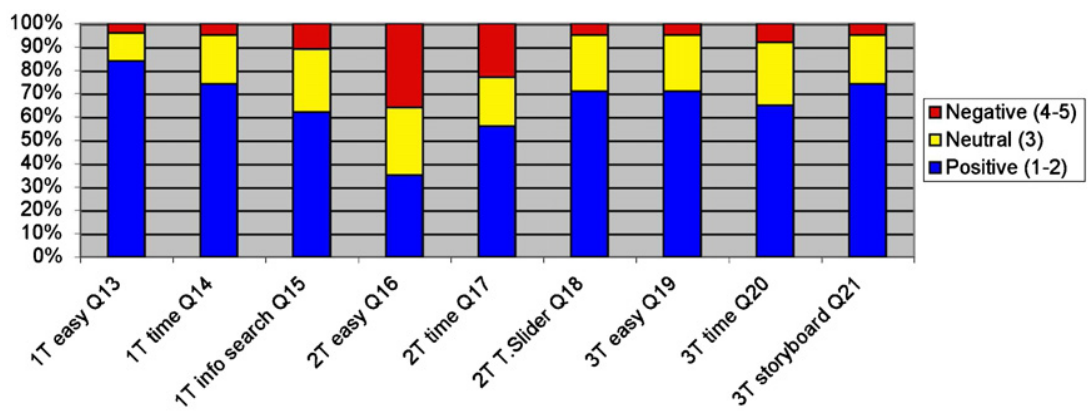

Fig. 15. Percentage of positive against negative answers on questions from Q13 to Q21. 


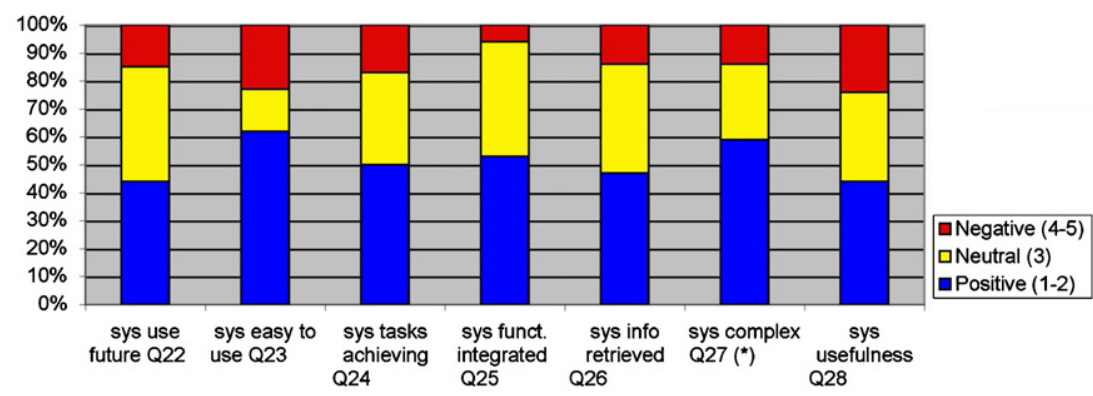

Fig. 16. The graph representing the percentage of positive against negative answers on questions from Q22 to Q28. Questions with (*) means that the question was posed in a negative but in the graph have been inverted to give an homogeneous overview.

Table 5

Average scores on questions from Q22 to Q28. Questions with a (*) mean that the question was posed negatively but has been inverted in the graph for a homogeneous overview.

\begin{tabular}{ll}
\hline Question & Average \\
\hline Q22 & 2.6 \\
Q23 & 2.5 \\
Q24 & 2.6 \\
Q25 & 2.5 \\
Q26 & 2.7 \\
Q27 $\left(^{*}\right)$ & 3.5 \\
Q28 & 2.7 \\
\hline
\end{tabular}

Table 6

Summary of experimental results.

\begin{tabular}{|c|c|}
\hline Positive & $\begin{array}{l}\text { Users liked the interface and the information organization } \\
\text { as well as the tutorial. The completion of proposed tasks } \\
\text { was good. The system resulted easy to use. Good } \\
\text { functionalities integration. }\end{array}$ \\
\hline Negative & $\begin{array}{l}\text { System stability (occasionally behaves in an unexpected } \\
\text { ways). Task } 2 \text { seemed difficult to complete. }\end{array}$ \\
\hline
\end{tabular}

${ }^{\text {a }}$ All but easy of completion of Task 2 (Q16).

The system is developed by using the web mashup techni que but, compared with other systems, it provides specia lized tools such as the spatio/temporal search feature, the recommendation and filtering tool and the storyboarding. Many social networks have been used during different types of emergencies like the Virginia Tech shooting or London bombings but they were general purpose like Facebook or Flickr; nevertheless, these systems resulted very helpful both during the emergency for keeping people in touch or update on the status of the emergency, and immediately after for recollecting data or tracing the events and com munications occurred during the emergency phase. Our system has been compared to others which includes similar information but lack of organization and tools helpful in such critical situations. We identified four dimensions: spatial, temporal, collaborative and situational that are common to mashup systems for emergencies. We categor ized the systems explored in the literature with these four dimensions and highlighted the characteristics and the limitations of each. We used the four dimensions to design our system for being as effective as possible being a georeferenced mashup system for back channels commu nications (based on images) for emergency situations. We evaluated our system by performing three different evalua tions: analytical, based on heuristic and experimental eva luation. From the evaluations, we found that users liked the interface, the information organization, and the system tutorial. Moreover, the system resulted easy to use and with good functionalities integration. This last characteristic is very good being our system a mashup and thus an integration of different sources of information and systems (google maps, flickr, and so on). On the other hand, users criticise the aesthetic of the interface which could be enhanced with their suggestions and the system resulted partially instable when tested on different browsers (this can be due to the peculiarities of the technologies which are always not completely standard when rendered in different browsers). The evaluation clearly shows the potential of our system and the efficacy in the presented scenarios. Further more, we think that our system is helpful both for people involved in an emergency (for retrieving information about relatives, for obtaining visual information about the status of an house or building, etc.) and for emergency profes sionals (a storyboard can be edited representing the photos indicating the status before, during and after an emergency, photos available before the emergency could be used to coordinate aids on site, etc.). Apart from improving the system according to users' evaluations, we are currently implementing new features to include in the mashup visualization additional information, such as 3D mappings produced by GoogleEarth ${ }^{22}$ augmented by carving Flickr photos onto the terrain space. Keywords or tags clustering is one of the features that could be of great help in our system as they can be considered a further dimension in the search for information [4]. We are also developing new tools for filtering photos of particular objects of interest like build ings, hospitals, and so on, as elements of interest for an emergency or disaster scenario joining image processing features with tags clustering. Finally, we are considering of integrating other media sources like text and videos taken from other social networks.

\footnotetext{
22 earth.google.com
} 


\section{Acknowledgements}

This work has been developed under UIA4SIGE (TSI2007 60388) and UrThey (TIN2009 09687) projects funded by Spanish Ministry of Science and Innovation. Prof. Levialdi's contribution to this work has been supported by a Chair of Excellence funded by Universidad Carlos III de Madrid and Banco Santander.

\section{Appendix A}

We present here the scenario and the three tasks of incremental difficulty proposed to the participants of our experimental evaluation, reported in Section 4.3.

\section{A.1. Scenario}

Hurricane Katrina in 2005 was one of the deadliest in the history of the United States. Among recorded Atlantic hurricanes, it was the sixth strongest overall. Hurricane Katrina formed over the Bahamas on August 23, 2005, and crossed southern Florida, causing some deaths and flood ing there before strengthening rapidly in the Gulf of Mexico. The storm weakened before making its second landfall on the morning of Monday, August 29 in south east Louisiana. It caused severe destruction along the Gulf coast from central Florida to Texas. The most severe loss of life and property damages occurred in New Orleans, Louisiana, which flooded as the levee system catastrophi cally failed, in many cases hours after the storm had moved inland. Use the eStoryS system to accomplish the following tasks, within the scenario presented above.

\section{A.1.1. First task}

Imagine you are writing about the hurricane Katrina in your personal blog, and you want to insert a picture in your post. Select one picture that, in your opinion, best describes the destruction caused by the passage of Katrina in New Orleans, Louisiana.

\section{A.1.2. Second task}

One of your best friends lives in Loyola Avenue, New Orleans, Louisiana. It is August 31, 2005 and you are worried about her/him because she does not answer the phone and stopped updating her/his blog. Search for photos taken in Loyola Avenue, New Orleans, Louisiana on the days August 2830,2005 , to check about the damages in that place that you believe are related to your friend's safety.

\section{A.1.3. Third task}

You are a member of the civil defense who, one month after the crisis, have to report on the passage of the hurricane, damages and recovery operations, document ing it with photos. Choose at least five and at most 10 pictures and build a sequence of such images to describe the situation in New Orleans, Louisiana, before the hurricane occurs (a few days before August 28, 2005), during the disaster (the week from August 28, 2005 to
September 4, 2005), and immediately after (let us say until three weeks after).

\section{A.2. Questionnaire design}

We devised our questionnaire after having screened a list of standardized questionnaires available in the litera ture. In particular we took into account the following instruments created to capture some aspects of usability criteria:

- Software Usability Measurement Inventory SUMI [18];

- Questionnaire for User Interaction Satisfaction QUIS [8];

- Purdue Usability Testing Questionnaire PUTQ [16];

- System Usability Scale SUS [5];

- After Scenario Questionnaire ASQ and Post Study System Usability Questionnaire PSSUQ [20].

\section{Appendix B. Supplementary data}

Supplementary data associated with this article can be found in the online version at doi:10.1016/j.jvlc.2010.12.003.

\section{References}

[1] P. Agouris, A. Stefanidis, in: Integrated Spatial Databases: Digita Images and GIS, Proceedings of International Workshop on ISD'99, vol. 1737, Springer, 1999.

[2] S. Ahern, M. Naaman, R. Nair, J.H.-I. Yang, World explorer: visualizing aggregate data from unstructured text in geo-referenced collections, in: JCDL '07: Proceedings of the Seventh ACM/IEEE Joint Conference on Digital Libraries, ACM, New York, NY, USA2007, pp. $1-10$.

[3] M.-A. Aufaure, C. Trpied, What approach for searching spatial information?, J Visual Lang. Comput. 12 (4) (2001) 351-373.

[4] A. Bellucci, S.L. Ghiron, I. Aedo, A. Malizia, Visual tag authoring: picture extraction via localized, collaborative tagging, in: S. Levialdi (Ed.), Proceedings of the Working Conference on Advanced Visual Interfaces, ACM Press2008, pp. 351-354.

[5] J. Brooke, System Usability Scale (SUS): A Quick-And-Dirty Method of System Evaluation User Information, 1986.

[6] W.A.S. Buxton. Chunking and phrasing and the design of humancomputer dialogues, in: Human-Computer Interaction: Toward the Year 2000, 1995, pp. 494-499.

[7] L. Carver, M. Turoff, Human-computer interaction: the human and computer as a team in emergency management information systems, Commun. ACM 50 (3) (2007) 33-38.

[8] J.P. Chin, V.A. Diehl, K.L. Norman, Development of an instrument measuring user satisfaction of the human-computer interface, in: CHI '88: Proceedings of the SIGCHI Conference on Human Factors in Computing Systems, ACM Press, New York, NY, USA1988, pp. 213-218.

[9] P. Currion, C. de Silva, B.V. de Walle, Open source software for disaster management, Commun. ACM 50 (3) (2007) 61-65.

[10] D.M. Boyd, N.B. Ellison, Social network sites: definition, history, and scholarship, J. Comput. Mediated Commun. 13 (1) (2007) 210-230.

[11] R. Dynes, Organized Behavior in Disaster, Heath Lexington Books, 1970.

[12] H.W. Fischer, Response to Disaster: Fact Versus Fiction and Its Perceptuation, second ed., University Press of America, New York 1998.

[13] D. Gillmor, We the Media: Grassroots Journalism by the People, for the People, O'Reilly Media, Inc., Sebastopol, CA, 2006.

[14] G. Haddow, J. Bullock, Introduction to Emergency Management Elsevier Science, USA, 2003.

[15] A.L. Hughes, L. Palen, J. Sutton, S.B. Liu, S. Vieweg, Site-seeing in disaster: an examination of on-line social convergence, in: Proceedings of the 2008 ISCRAM Conference, Washington, 2008. 
[16] H.X. Lin, Y-Y. Choong, G. Salvendy, A proposed index of a usability method for comparing the relative usability of different software systems, Behav. Inf. Technol. 16 (1997) 267-278.

[17] L. Kennedy, M. Naaman, S. Ahern, R. Nair, T. Rattenbury, How flickr helps us make sense of the world: context and content in community-contributed media collections, in: MULTIMEDIA '07: Proceedings of the 15th International Conference on Multimedia, ACM, New York, NY, USA2007, pp. 631-640.

[18] J. Kirakowski, M. Corbett, Sumi: the software usability measurement inventory, Br. J. Educ. Technol. 3 (1993) 210-212.

[19] Y.G. Leclerc, M. Reddy, L. Iverson, A. Heller, The geoweb: a new paradigm for finding data on the web, in: Proceedings of the International Cartographic Conference (ICC2001), Beijing, 2001.

[20] J.R. Lewis, Ibm computer usability satisfaction questionnaires: psychometric evaluation and instructions for use, Int. J. Hum.Comput. Interact. 7 (1) (1995) 57-78.

[21] R. Likert, A technique for the measurement of attitudes, Working Paper, 1932.

[22] G. Linden, B. Smith, J. York, Amazon.com recommendations: itemto-item collaborative filtering, Internet Comput. IEEE 7 (1) (2003) 76-80.

[23] S.B. Liu, L. Palen, J. Sutton, A.L. Hughes, S. Vieweg, In search of the bigger picture: the emergent role of on-line photo sharing in times of disaster, in: ISCRAM '08: Proceedings of the Fifth International ISCRAM Conference, 2008 , pp. 140-149.

[24] J. Nielsen, Guerrilla HCI: using discount usability engineering to penetrate the intimidation barrier, in: R.G. Bias, D.J. Mayhew (Eds.),
Cost-justifying Usability, Morgan Kaufmann Publishers, Burlington, MA, 1994, pp. 245-272.

[25] L. Palen, S.B. Liu, Citizen communications in crisis: anticipating a future of ict-supported public participation, in: $\mathrm{CHI}$ '07: Proceedings of the SIGCHI Conference on Human Factors in Computing Systems, ACM, New York, NY, USA2007, pp. 727-736.

[26] J. Preece, D. Benyon, O. University, A Guide to Usability: Human Factors in Computing, Addison-Wesley Longman Publishing Co., Inc., Boston, MA, USA, 1993.

[27] W.A. Schafer, C.H. Ganoe, J.M. Carroll, Supporting community emergency management planning through a geocollaboration software architecture, Comput. Supported Coop. Work 16 (4-5) (2007) 501-537.

[28] B. Shneiderman, C. Plaisant, Designing the User Interface, fourth ed., Addison-Wesley Publishing Company, Reading, MA, 2005.

[29] J. Sutton, L. Palen, I. Shlovsk, Back-channels on the front lines: emergent uses of social media in the 2007 southern california wildfires, in: Proceedings of the 2008 ISCRAM Conference, Washington, 2008.

[30] K.J. Tierney, M.K. Lindell, R.W. Perry, Facing the Unexpected: Disaster Preparedness and Response in the United States, John Henry Press, Washington, DC, 2001.

[31] K. Toyama, R. Logan, A. Roseway, Geographic location tags on digital images, in: MULTIMEDIA '03: Proceedings of the 11th ACM International Conference on Multimedia, ACM, New York, NY, USA2003, pp. 156-166.

[32] A. Voisard, Mapgets: a tool for visualizing and querying geographic information, J. Visual Lang. Comput. 6 (4) (1995) 367-384. 